\title{
Comparison of the Medtronic SelectSecure and Conventional Pacing Leads: Long Term Follow-up in a Multicenter Pediatric and Congenital Cohort
}

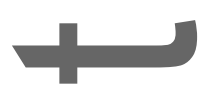

Jeffrey P. Moak, MD ${ }^{1}$, lan H. Law, MD², Martin J. LaPage, $\mathrm{MD}^{3}$, Frank Fish, $\mathrm{MD}^{4}$, Ira Shatty, $\mathrm{MD}^{5}$, Anne M. Dubin, $M D^{6}$, Akash Patel, $M D^{7}$, Peter Fishbach, $M D^{8}$, Nicole Cain, $M D^{9}$, Christopher Johnsrude, $\mathrm{MD}^{10}$, Charles I. Berul, $\mathrm{MD}^{1}$, Aminata Bangoura, $\mathrm{BS}^{1}$, Sridhar Hanumanthaiah, MBBS, $\mathrm{MS}^{1}$, Robert McCarter, $\mathrm{SCD}^{11}$

${ }^{1}$ Division of Cardiology, Children's National Health System, Washington, DC

${ }^{2}$ Division of Pediatric Cardiology, University of lowa Stead Family Children's Hospital, lowa City, IA

${ }^{3}$ Division of Cardiology, University of Michigan, Ann Arbor, MI

${ }^{4}$ Division of Cardiology, Vanderbilt University, Nashville, TN

${ }^{5}$ Division of Cardiology, Advocate Children's Hospital, Oak Lawn, IL

${ }^{6}$ Division of Pediatric Cardiology, Stanford University, Palo Alto, CA

${ }^{7}$ Division of Cardiology, University California, San Francisco, CA

${ }^{8}$ Division of Cardiology, Sibley Heart Center, Atlanta, GA

${ }^{9}$ Division of Cardiology, Medical University of South Carolina, Charleston, SC

${ }^{10}$ Division of Cardiology, University of Louisville, Louisville, KY

${ }^{11}$ Division of Biostatistics and Study Methodology, Children's National Health System, Washington, DC

Abbreviated Title: Medtronic SelectSecure Pacing Lead

Funding: Medtronic, Minneapolis, MN

Conflicts of Interest: None to disclose for all authors

Address for correspondence:

Jeffrey P. Moak, MD

Division of Pediatric Cardiology

Children's National-Health System

This is th auth $\mathrm{r}$ manuscript accepted for publication and has undergone full peer review but has not been throug - copyediting, typesetting, pagination and proofreading process, which may lead to differences between this version and the Version of Record. Please cite this article as doi:

10.1111/pace.13614.

This article is protected by copyright. All rights reserved. 
111 Michigan Ave, NW

Washington, DC 20010

Email: jmoak@childrensnational.org

202-476-2020(P)

202-476-5700(F)

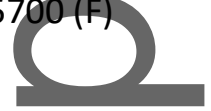

Abstract

Introduction: The Medtronic SelectSecure ${ }^{\mathrm{TM}}$ pacing lead (SS) has theoretical advantages compared to conventional $(\mathrm{C})$ transvenous pacing leads $(\mathrm{PL})$. The study purpose was to determine whether differences in electrical function and lead survival exist between these PL in a large data set of pediatric and congenital patients.

Methods: A multicenter historical longitudinal cohort study was performed comparing SS and CPL performance over a 72-month follow-up (FU). Ten centers provided data for both SS and CPL, matched for age, implanted pacing chamber, time period of implantation, and presence of heart disease

Results: The cohort consisted of 141 subjects in each group. No statistical differences were observed in age, gender, presence of heart disease, or pacing indication. Atrial and ventricular capture thresholds were stable throughout FU and higher in the SS group (atrial: $0.75 \pm 0.02$ vs. $0.5 \pm 0.04 \mathrm{~V}$, ventricular; $1.0 \pm 0.04$ vs. $0.75 \pm 0.04 \mathrm{~V}), \mathrm{p}<0.001$. Group PL sensing thresholds did not differ. The SS group required greater energy to pace (atrial: $0.57 \pm 0.05$ vs. $0.32 \pm 0.02 \mathrm{~mJ}$, ventricular: $0.83 \pm 0.05$ vs. $0.56 \pm 0.06 \mathrm{~mJ}), p=0.001$. Early lead dislodgement and phrenic nerve stimulation were greater in the SS group $(p=0.03)$. Long-term lead survival was high and similar between the two groups, $p=0.35$. Conclusions: Long-term survival of both PL was high with a low fracture rate. The SS had excellent electrical function but did show higher capture thresholds and increased energy to pace; these differences are offset by other advantages of the SS PL.

This article is protected by copyright. All rights reserved. 


\section{Key Words:}

Transvenous pacing

Medtronic SelectSecure pacing lead

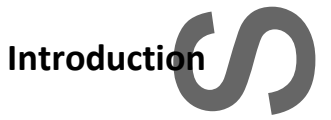

Technologic development of cardiac pacemakers has advanced to such a high level of manufacturing precision that very few failures of the pulse generator are seen clinically today. At present, the weakest link in the cardiac pacing system continues to be the pacing leads. Historically, older generation transvehous pacing leads were fraught with high capture thresholds, sensing failure, insulation breaks and conductor fractures. ${ }^{1-6}$ Previous studies reported transvenous pacing lead fracture rates in children between $5 \%$ and $17 \%$ over a follow-up of 2-5 years. ${ }^{1-6}$ Newer generation steroid eluting pacing leads have markedly decreased the frequency of subacute capture and sensing failure. ${ }^{5}$ However, the long-term survival of these pacing leads is still not described.

Pacing lead malfunction can result from lead fracture (either in the insulation coating or the metal conductor), high capture thresholds, poor sensing characteristics, lead dislodgement or lead stretch from growth in children. ${ }^{2}$ Lead extraction and replacement is often required secondary to lead (1) malfunction, need to upgrade from a pacing to ICD system, patient growth or infection. Removal of most currently available chronic pacing leads is complex, requiring expertise in pacing lead extraction with specialized tools. ${ }^{7,8}$

This article is protected by copyright. All rights reserved. 
Theoretically, the structural build of the Medtronic SelectSecure (Model 3830) (Minneapolis, MN) transvenous pacing may promote longevity and freedom from lead fracture, as well as ease of removal after development of lead malfunction. ${ }^{9}$ The SelectSecure pacing lead (4.1 Fr. outer lead body diameter) is (umenless and is composed of a MP35N conductor with hybrid insulation (polyurethane outer and silicone inner). The lead has a beclomethasone eluting-collar to improve pacing and sensing thresholds. The lack of a central lumen and the use of a conductor cable, rather than coil, allows for increased insulation redundancy, high tensile strength, and reduced bulk.

\section{ऽ}

This study hypothesized that this novel lumenless design would result in 1) improved lead survival, 2) equivalent sensing and capture characteristics, and 3) less complicated lead extraction compared to stylet-driven traditional transvenous pacing leads.

\section{Methods}

Study Population: Ten pediatric electrophysiology centers participated in a historical parallel group longitudinalmulticenter cohort study design to compare the performance of the lumenless SelectSecure (Model 3830) pacing lead with conventional active fixation stylet-driven pacing leads. Participating centers were chosen to have substantial experience in the techniques required for implanting the SelectSecure pacing lead, and also to have, in the same era, implanted conventional non-SelectSecure pacing leads. In total, 141 SelectSecure and 141 conventional pacing leads were used in our data analysis.

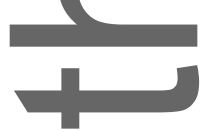

All conventional pacing leads were required to have a bipolar configuration, steroid eluting lowthreshold electrode tip design, and active fixation mechanism, Table 1. An attempt was made to match the SelectSecure and conventional pacing leads for subject age at implantation (+/- 8 years), 
chamber of lead implantation and the presence/absence of congenital heart disease, at each center.

Each center implanted both SelectSecure and conventional pacing leads.

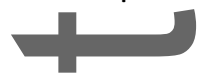

Each participating institution obtained IRB approval for enrolling subjects in this study. All data were de-identified and entered into a password-protected RedCap database, maintained at Children's National Health System.

\section{Data Collection:?}

Baseline data included: 1) subject demographics (date of birth, gender, race), 2) date of implant procedure, 3) pacing lead manufacturer and model number, 3) site of pacing lead position (atrial or ventricular, and location in the chamber if known), 4) electrophysiologic indication for pacing lead implantation, 5) presence and type of heart disease. Lead initial performance data included 1) capture threshold (Volts), 2) sensing threshold (mV), 3) lead impedance (Ohms), and 4) complications at the time of implantation or within the first 30 days after implantation.

Follow-up data collected every 12 months to the maximum time of follow-up included: 1) date of<smiles>[CH]1[CH]C1</smiles>
follow-up, 2) capture threshold (Volts), 3) paced pulse width, 4) sensing threshold (mV), 5) lead impedance (Ohms)and 6) complications during follow-up. Complications included: 1) cardiac perforation, 2) failure to capture, 3) failure to sense, 4) lead dislodgement, 5) phrenic nerve stimulation, 6) lead,fracture or insulation breach with lead impedance out of range, and 7) venous (1) thrombosis. If the implanted pacing lead needed revision, either for malfunction or upgrade (e.g. to an ICD lead), datawere collected on whether the pacing lead was retained and abandoned, or extracted. Extraction data collected included date of extraction, ease of extractability, method used for extraction, and any complications resulting from lead extraction. 
Pacing Energy Calculation: The energy required for cardiac pacing was calculated from simultaneously measured pacing lead capture threshold, pulse width of stimulation and pacing lead impedance. Energy to pace was calculated for each subject at each time point of follow-up. The following formula was used to calculate the pacing energy at capture threshold: Energy = ( (Voltage of stimulation $)^{2}$ XPulse Width)/ Impedance. Pacing energy was determined at a pulse width of 0.5 msec.

\section{क}

Statistical Analysis: Analyses proceeded from baseline comparisons of comparability by lead study group (SelectSecure vs. conventional pacing lead) to longitudinal modeling allowing for comparative evaluation of electrical performance, complication and extraction rates over time. Before conducting analyses of pacing lead electrical performance, the distribution of acute and chronic measurements was evaluated to determine whether the normality assumption was met to permit

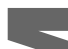
use of linear regression models. The distributions were not normally distributed and traditional data transformations failed to satisfy the normality assumption, $p<0.001$. Therefore, parametric modeling was rejected, and quantile regression analysis based on qreg in Stata 15 was used instead to estimate medians rather than means, which would be subject to greater influence of measurements that depart from normality. ${ }^{10}$ Time-to-event analyses based on Cox proportional hazards modeling was used to compare lead survival, freedom from failure and complications, and need for lead extraction. Both models controlled for differences between groups at baseline and of duration of follow-up, including evaluation of interactive effects of lead group by follow-up time. Analyses accounted for correlation due to matching of patients receiving SelectSecure and conventional pacing leads, as well as correlation due to multiple assessments of the same subject 
over time. Two-tailed p-values below 0.05 were considered statistically significant. Data were reported as median \pm standard error.

Results
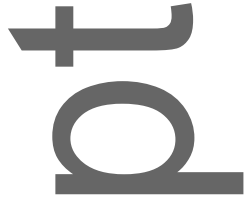

Study population: The study cohort consisted on 141 subjects implanted with the SelectSecure pacing lead, and 141 patients in the conventional population. Demographics are shown in Table 2. As expected, no difference in age, gender, ethnicity or presence of congenital heart disease (Table 3) was found when comparing the two groups. No significant differences were found in the indication for pacing lead implantation between the two groups (Table 4). No difference in follow-up time between the two groups ( $58 \pm 2.2$ vs. $57.7 \pm 2.1$ months, $p=0.92$ ) was seen. SelectSecure pacing leads were implanted between January 2006 and September 2014.The conventional pacing leads were implanted between November 1999 and September 2014, with substantial overlap between the two pacing lead groups from 2006 to 2014. Study subjects ranged in age from 3 to 36 years of recting age, median ages in the SelectSecure and conventional pacing lead groups were 13.7 vs. 14.1 years, respectively, $p=0.58$.

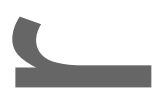

Conventional Pacing Lead Types: The manufacturer and model number for the conventional pacing leads are tabulated in Table 1 . The majority (89\%) were manufactured by Medtronic (Minneapolis, MN), and consisted of Model Numbers 4076 and 5076.

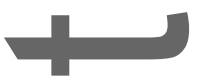

Pacing Lead Position: The SelectSecure and conventional pacing lead groups were composed of similar number of implanted pacing leads in either the atrium or ventricle and in both chambers, 65 , 32, 44 (SelectSecure group), and 66, 29, 46 (conventional group), respectively, $p=0.94$ 
Sites for atrial lead placement in the SelectSecure pacing group were the right atrial lead appendage (47), right atrial free wall (28), right atrial septum (13), left atrium (4), and not specified (17). Sites for atrial lead placement in the conventional pacing lead group were right atrial lead appendage (61), right atrial free wall (18), right atrial septum (4), left atrium (4), and not specified (25). SelectSecure atrial pacing leads were placed more frequently at sites other than the right atrial appendage (49\%-vs. $30 \%), p=0.03$.

Sites for ventricular lead placement in the SelectSecure pacing group were the right ventricular apex (19), right ventricular outflow tract (5), right ventricular septum (35), right ventricular free wall (2), left ventricle (2), and not specified (13). Sites for ventricular lead placement in the conventional pacing lead group were right ventricular apex (30), right ventricular outflow tract (0), right ventricular septum (30), right ventricular free wall (4), left ventricle (2), and not specified (9). SelectSecure-ventricular pacing leads were placed more frequently at sites other than the right atrial apex (70\% vs. $55 \%), p=0.02$.

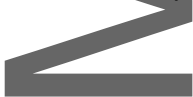

\section{Pacing Lead Electrical Performance}

Acute Measurements:

Acute Atrial and Ventricular Capture Thresholds: No significant differences were noted between the SelectSecure and the conventional pacing lead groups in the median acute atrial $(0.70 \pm 0.06 \mathrm{vs.} 0.60$ \pm 0.04 Volts, $p=0.14)$, and ventricular $(0.46 \pm 0.05$ vs. $0.55 \pm 0.04$ Volts, $p=0.15)$ capture thresholds. ren

Acute Atrial and Ventricular Sensing Thresholds: No significant differences were noted between the SelectSecure pacing lead and the conventional pacing lead groups in the median acute atrial (3.3 \pm 8

This article is protected by copyright. All rights reserved. 
0.23 vs. $3.2 \pm 0.21 \mathrm{mV}, \mathrm{p}=0.73)$ and ventricular (10.5 \pm 0.89 vs. $9.8 \pm 0.64 \mathrm{mV}, \mathrm{p}=0.69)$ sensing thresholds.

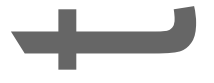

Acute Atrial and Ventricular Pacing Lead Impedance Measurements: Acute atrial lead impedance was significantly higher in the SelectSecure $(679.2 \pm 23.2 \mathrm{ohms})$ compared to the conventional pacing lead group $(614.9 \pm 22.0 \mathrm{ohms}, \mathrm{p}=0.04)$. Acute ventricular pacing lead impedance did not differ between the SelectSecure $(744.6 \pm 23.0 \mathrm{ohms})$ and the conventional pacing lead group $(727.1$ \pm 30.8 ohms, $p=0.69)$. Impedance measurements were within the normal operative range $(<200$ or $>$ 2000 ohms)

\section{Chronic Measurements:}

Chronic Atrial and Ventricular Capture Thresholds (> 1-month Follow-up): Median chronic atrial and ventricular pacing lead capture threshold measurements remained stable over a 72-month follow-up period, Figures $1 \mathrm{~A}$ and $1 \mathrm{~B}$. Chronic atrial and ventricular pacing thresholds were higher in the SelectSecure compared with the conventional pacing lead group, $p<0.001$ and $p<0.001$, respectively. Time-averaged chronic atrial pacing thresholds for the SelectSecure pacing leads were $0.75 \pm 0.02 \mathrm{~V}$ Vs. conventional pacing lead group $0.5 \pm 0.04 \mathrm{~V}, \mathrm{p}<0.001$. Time-averaged chronic ventricular pacing thresholds for the SelectSecure pacing leads were $1.0 \pm 0.04 \mathrm{~V}$ vs. the conventional pacing lead group $0.75 \pm 0.04 \mathrm{~V}, \mathrm{p}<0.001$. Time-averaged values were integrated over a 72-month period of follow-up. Voltage threshold was determined at $0.5 \mathrm{msec}$.

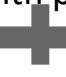

Chronic Atrial and Ventricular Sensing Thresholds (> 1-month Follow-up): Median chronic atrial and ventricular pacing lead sensing threshold measurements were stable over a 72-month follow-up period and did not differ between the SelectSecure pacing lead and conventional groups, $p \geq 0.98$.

This article is protected by copyright. All rights reserved. 
Chronic Atrial and Ventricular Pacing Lead Impedance Measurements (> 1-month Follow-up): None of the SelectSecure pacing leads had an impedance considered out of operative range $(<200$ or $>$ 2000 ohms). The pattern of changes in chronic atrial pacing lead impedance measurements over time differed between the SelectSecure and conventional pacing lead groups, $p=0.016$, figure $2 \mathrm{~A}$. Chronic atrial pacing lead impedance decreased over time in the SelectSecure group and remained stable in the conventional atrial pacing lead group. Impedance measurements were within the normal operative range ( $<200$ or $>2000$ ohms).

Likewise, changes in chronic ventricular pacing lead impedance measurements over time differed
between the SelectSecure and conventional pacing lead groups $(p=0.045)$, figure $2 B$. As with atrial pacing leads, chronic ventricular pacing lead impedance decreased over time in the SelectSecure group and decreased much more slowly in the conventional ventricular pacing lead group.

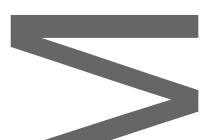

These differences are reflected in the time-averaged impedance measurements for the atrial and ventricular pacing lead groups, which were higher in the SelectSecure group vs. the conventional pacing lead group during a 72-month period of observation. Time-averaged impedance measurements for the atrial pacing lead groups were higher in the SelectSecure group (523.4 \pm 6.2 Ohms) vs. the conventional pacing lead group (476.1 \pm 6.7 Ohms), $p<0.05$. Time-averaged ventricularlead impedance measurements were higher in the SelectSecure group $(550.0 \pm 8.1$ 1

Ohms) vs. the conventional pacing lead group (486.6 \pm 8.3 Ohms), $\mathrm{p}<0.05$.

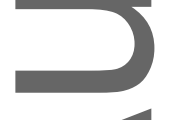

Energy for Chronic Atrial and Ventricular Pacing (>1-month Follow-up): Changes in calculated atrial pacing lead energyremained stable over time in both groups and differed between the SelectSecure 
and conventional pacing lead groups, $p<0.001$, figure 3A. Time-averaged chronic atrial pacing lead energy was higher in the SelectSecure than in the conventional pacing lead group, $0.57 \pm 0.05$ vs.

$0.32 \pm 0.02 \mathrm{~mJ}$, respectively, $\mathrm{p}<0.001$

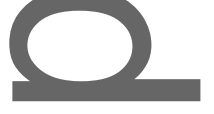

Long-term changes in calculated ventricular pacing lead energy increased slightly over time in both groups ( $p=0.048$ ) and differed between the SelectSecure and conventional pacing lead groups, $p=0.045$, figure $3 B$. The time-averaged chronic ventricular pacing lead energy in the SelectSecure group was higher than observed in the conventional pacing lead group, $0.83 \pm 0.05$ vs. $0.56 \pm 0.06$ $\mathrm{mJ}$, respectively, $\mathrm{p}=0.001$.

\section{Pacing Lead-Related Complications.}

Early Complications Occurring at Time of Pacing Lead Implantation and During the First Month of Follow-up: Complications occurred more commonly in the SelectSecure group in the first month following pacing lead implantation (19 vs. 8, p=0.03). Complications included lead dislodgement ( 7 vs. 2), lead'injury during sheath splitting (1 vs. 0), phrenic nerve stimulation (8 vs. 2), pericardial effusions ( 1 vs. 3), ventricular oversensing ( 2 vs. 0), and pneumothorax (0 vs. 1 ).

Late Pacing Lead Complications Occurring After 1-month of Follow-up: Long-term complications during pacing lead follow-up did not differ between the SelectSecure (8) and the conventional pacing lead group (10), $p=0.35$, Table 5 .

The complication rate per 1000-person months of follow-up was not statistically different between the SelectSecure $(0.78 ; 95 \% \mathrm{Cl}=0.35$ to 1.74$)$ and the conventional pacing lead group $(1.11 ; 95 \% \mathrm{Cl}=$ 0.58 to $2.121, p=0.26$

This article is protected by copyright. All rights reserved. 
Pacing Lead Survival Analysis: No difference was noted in the follow-up rate of lead complications up to 72 months of follow-up, $p=0.50$. The overall complication rate was low, 0.95 per 1000-person months of follow-up. A Kaplan-Meier plot of pacing lead survival free from complications was shown in figure 4.

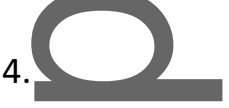

-

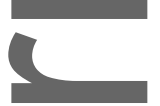

Pacing lead survival was separately analyzed by need for pacing lead extraction. While the SelectSecure pacing lead group showed a modest increase in risk for undergoing lead extraction (hazard ratio $=1.28$ ), this was not statistically different from the conventional pacing lead group $(p=0.70)$.

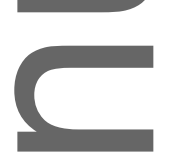

Pacing Lead Extraction: Sixteen pacing leads were extracted, 11 SelectSecure and 5 conventional pacing leads. The conventional leads were manufactured by Medtronic (model 5076, $n=3$, and 4076, $n=2$ ). Extraction in the SelectSecure lead group was performed using simple traction (10) and a laser sheath (1). Indications for lead extraction were dislodgement (9), oversensing (1), and phrenic nerve stimulation (1). Average implant duration was $18 \pm 21$ months. Indications for pacing lead extraction in the conventional group were fracture (2), failure to capture (2) and non-specified malfunction (1). Average implant duration was $36 \pm 27$ months. Extraction was performed using simple traction (3) and a laser sheath (2). No complications occurred during any of the extraction procedures.

\section{Discussion}

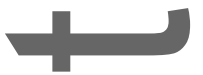

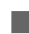

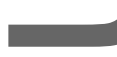

The data from this study confirm previous reports that the SelectSecure pacing lead has excellent acute and Tong-term capture and sensing thresholds in both the atrium and ventricle ${ }^{9,11-16}$ Two other studies have reported on the long-term changes in capture and sensing thresholds and lead 
impedance changes in SelectSecure leads. ${ }^{11,13}$ Bansal et al found statistically significant decreases in atrial capture threshold, atrial lead impedance and ventricular lead impedance. However, significant increases in ventricular capture threshold were found. Chronic atrial and ventricular sensing thresholds were stable. In contrast, Garnreiter et al reported somewhat different long-term changes in atrial and ventricular capture and sensing thresholds. ${ }^{11}$ Statistically significant increases in atrial and ventricular capture thresholds, P wave and R wave amplitudes were noted. Statistically significant decreases in the atrial and ventricular lead impedance measurements were appreciated. Collectively, these two studies along with findings of this study revealed a consistent decrease in atrial and ventricular lead impedances over time. The data from this study reconcile the directional opposite changes in atrial and ventricular capture and sensing thresholds reported between Bansal et al. ${ }^{13}$, and Garnreiter et al. ${ }^{11}$ Subsequent to 1 month of follow-up chronic atrial and ventricular capture and sensing thresholds were stable in the SelectSecure pacing lead group.

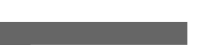

What might be the long-term implications of the higher capture threshold and increased pacing lead impedance for the SelectSecure pacing lead group? Despite having a higher chronic pacing lead impedance, which can be advantageous in decreasing the energy to pace, we observed a larger longitudinal decrease in pacing lead impedance in the SelectSecure group compared with the conventional pacing lead group. The SelectSecure pacing lead group also demonstrated a consistently elevated capture threshold compared with the conventional pacing lead group, which may shorten battery life. Calculated energy to pace using simultaneously measured capture thresholds, pulse width and lead impedances (figure 3 A and B), suggested that the SelectSecure pacing lead may increase pacing energy requirements, thereby potentially reducing pacemaker battery longevity. Pacemaker longevity projections were performed using each pacing lead system (SelectSecure and conventional pacing leads) in three different pacing scenarios (data not shown): 1)

This article is protected by copyright. All rights reserved. 
VVI pacing at $50 \%, 2$ ) VVI pacing at $100 \%$, and 3) DDD pacing (atrial pacing - $25 \%$ and ventricular pacing $-100 \%)$. A two-fold safety margin was programmed into the longevity calculator. The weighted average heart rate over the age range of our study population was calculated at $85 / \mathrm{min}$. The pacemaker used for the calculations had one of the most efficient internal circuitry energy drains. The SelectSecure pacing lead group in the DDD model had a shorter mean device survival by 0.7 years, and in the VVI paced $100 \%$ model a shorter mean survival by 0.6 years, reinforcing the possible clinical importance of the data provided regarding higher pacing energy requirements in the SelectSecure pacing group. To put this into perspective, we reviewed literature on the extension of device longevity promoted using software developments aimed at maximizing battery longevity Capture Management (minimizing voltage output) and AV search hysteresis (limiting ventricular pacing). ${ }^{17,18}$ Average extension of device longevity by these advanced pacing algorithms was between $6-8$ months, a difference similar in magnitude to the decrease in device longevity associated with the SelectSecure pacing lead compared with the conventional pacing lead group.

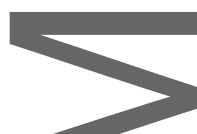

The SelectSecure pacing lead provided flexibility for alternative site pacing; $49 \%$ of the SelectSecure atrial pacing leads and $70 \%$ of SelectSecure ventricular pacing leads were implanted outside of the right atrial appendage for the atrial lead and outside of the right ventricular apex for the ventricular lead. Flexibility in lead site placement may be preferable in patients with congenital heart disease in whom the right atrial appendage anatomy may be distorted after open heart surgery or when considering alternative sites for lead placement to help improve cardiac hemodynamics. Karpawich

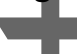
et al highlighted the importance of the site of right ventricular pacing on paced ventricular function and being able to implant the ventricular pacing lead at alternative sites outside the right ventricular apex. ${ }^{19}$ His bundle pacing using SelectSecure pacing leads is becoming an alternative to traditional biventricular pacing and is another example of the importance of this concept. ${ }^{20}$ 
The SelectSecure pacing lead had very favorable long-term survival, with an estimated failure rate of 0.78 per 1,000-person months of patient follow-up. Lead fracture was extremely uncommon (2/141 pacing leads) in either group. This finding was in contrast to older case series of long-term pacing lead survival in pediatric patients which have suggested vulnerability of transvenous pacing leads to lead fracture, Olgun et al. noted a 7.3\% transvenous pacing lead failure rate over a 5-year follow-up period. ${ }^{1}$ Most of the lead fractures occurred in active fixation leads. Fortescue et al noted multiple modes for failure of transvenous pacing leads - insulation break, lead fracture, high thresholds, stretch and late dislodgement with an actuarial survival of only $82 \%$ at 5 years. ${ }^{2}$ Silvetti et al. reported their experience with endocardial pacing in neonates and infants and noted a $10 \%$ failure rate (one early dislodgement and 1 late threshold rise). ${ }^{3}$

Few studies have compared the performance of the SelectSecure pacing lead with conventional design stylet-driven pacing leads. Garnreiter et al. compared the performance of SelectSecure pacing leads against a thin transvenous stylet-driven pacing lead, the St. Jude model 1488 active fixation steroid eluding bipolar pacing lead. ${ }^{11}$ The average length of follow-up was $26+/-19$ months. Nine complications (5\%) occurred in the SelectSecure pacing lead group (poor capture threshold -4 , lead dislodgement -3 , pocket infection -1 , and phrenic nerve stimulation -1 ). In contrast, $20 \%$ of the control St Jude model 1488 pacing leads had complications - lead fracture (12), poor capture threshold (4), lead noise (2), lead dislodgement (1), and extracardiac pacing (1). Similar to the data reported by Garnreiter et al. ${ }^{11}$, overall lead survival of the SelectSecure lead in this study did not differ from the conventional pacing lead group. In the report by Garnreiter et al, freedom from atrial lead complications (SelectSecure and conventional pacing leads) over 5 years was between 95 and $90 \%$, and for ventricular leads (SelectSecure and conventional pacing leads) was around $90 \% .{ }^{11}$ In 
this study the freedom from lead complication at 5 years in the SelectSecure pacing lead population was $96 \%$ and for the conventional pacing lead group was $94 \%$. These results suggest the robustness of the SelectSecure lead design as well as improvement in the reliability of the conventional pacing leads compared to data reported on older pacing lead designs.

Of note, however, lead dislodgement was found more frequently in the SelectSecure (11/141) pacing lead group Lead dislodgment was reported by five of the ten participating centers. There are several explanations for this. This may be related to the complexities of using an implant sheath and sheath splitter. Centers reporting lead dislodgement were independently queried, and thought that lead dislodgement occurred early in their experience and involved structural heart disease cases. Lack of adequate lead slack resulted in late dislodgement secondary to lead stretch from patient growth. Early lead dislodgement may be lessened using some lessons reported by Redfearn et al. ${ }^{21}$ and Shali et al. ${ }^{22}$ Using a rabbit animal model for testing electrical characteristics that might predict successful pacing lead implantation, Shali et al showed that monitoring the current of injury response to lead implantation provided insight into lead stability. ${ }^{22}$ The magnitude (amplitude of ST segment elevation) and the duration of persistence of the current of injury predicted whether the lead was just in contact with the myocardium, partially or fully rotated and advanced into the heart tissue. Current of injury persisted longer for leads fully rotated and advanced into the myocardium compared to partially rotated leads ( $26.5 \pm 62.8 \mathrm{~min}$ vs. $5.6 \pm 2.0 \mathrm{~min}, \mathrm{p}, 0.05)$. Paying more attention to the implant electrogram dynamics, particularly the current of injury, may provide the implanter ror with better insight into whether the SelectSecure pacing lead tip has been fully rotated into the myocardium, thereby potentially decreasing the likelihood of lead dislodgement.

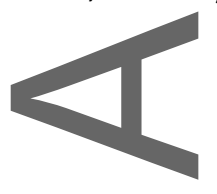

This article is protected by copyright. All rights reserved. 
While the numbers were small in this study, the SelectSecure pacing lead was easily extracted using simple traction in 10 of 11 patients, suggesting an advantage for implanting this lead in case of future need for pacing lead extraction secondary to lead failure or need for lead substitution. In the study reported by Garnreiter et al., nine SelectSecure pacing leads required extraction. ${ }^{11}$ All were successfully extracted using manual traction with counterclockwise rotation. From a population of 22 attempted SelectSecure pacing lead extraction procedures, Shephard et al. were able to extract 9 SelectSecure pacing leads using simple traction alone; however, 7 required the use of polypropylene sheaths and 6 needed a cutting sheath; average lead implant time was 4.1 years. ${ }^{8}$ Early data suggest an advantage for the SelectSecure pacing lead in facilitating easier lead removal if needed.

This study revealed the high reliability of pacing in children using either the SelectSecure pacing lead or the conventional pacing lead group. Freedom from complications was similar between the groups. The mechanism for lead failure however differed between the two groups. Implantation of the SelectSecure pacing lead required a technique different from stylet-driven pacing leads. The more technically advanced challenges of implanting the SelectSecure pacing lead resulted in a higher early dislodgment rate, which has also been reported in previous studies. ${ }^{11,12,16}$ Of note, a low lead fracture rate was seen in both the SelectSecure and the conventional pacing lead groups.

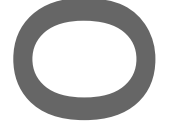

What potential attributes can be ascribed to the SelectSecure pacing lead? The SelectSecure pacing lead did provide flexibility in positioning the lead in alternative sites for pacing, and ease of extraction. Alternative sites of pacing can have definite hemodynamic advantage. Pacing lead survival rate was high with a low fracture rate. The SelectSecure pacing lead demonstrated excellent electrical function. Not assessed in this study were differences between the SelectSecure and conventional pacing lead groups on tricuspid valve function and venous thrombosis. Data from 
Bharmanee et al. would suggest an advantage for the SelectSecure pacing lead in allowing better atrioventricular valve function and less likelihood of venous compromise. ${ }^{23}$

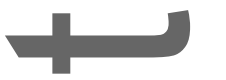

Limitations: Study limitations include historical data collection based primarily on record review. This study was not a randomized comparison of the SelectSecure pacing lead with conventional stylet-driven pacing leads or any specific stylet-driven lead design. The choice of which lead to implant in any patient was up to the discretion of the operator and introduces potential biases. However, the study design was not intended to compare the SelectSecure pacing lead with a specific conventional style-driven pacing lead model, but a group of the usual conventional stylet-driven pacing leads deployed in pediatric pacing practice. The authors felt it unfair to restrict the conventional group to a specific lead design in which data would be parsed introducing other bias choosing lead designs with superior or inferior performance compared with the SelectSecure group. In reality, functional pacing lead outcomes are not merely dependent on a specific pacing lead build, infliencerd a

but are influenced as much by other factors such as 1) patient characteristics - presence of heart disease, type of heart disease, patient age at implant, 2) chamber of implantation, and 3) operator/implant center characteristics. To account for these other influences, each SelectSecure pacing lead from each center was matched with a conventional pacing lead implanted in a comparable aged-subject, matched by presence or absence and type of associated heart disease, matched by the cardiac chamber of implantation - atrium or ventricle from that center, and matched by "era" of implantation.

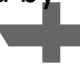

Conclusions: The SelectSecure pacing lead did provide flexibility in positioning the lead at alternative sites for pacing, and ease of extraction. Since the SelectSecure pacing lead lumenless design is newer, the higherpacing lead dislodgement rate in the early post-implantation period may be 
related to a learning curve inherent to its deployment. Long-term pacing lead survival and complication-free survival in both the SelectSecure and conventional pacing lead groups was high with a low fracture rate, which is different from previously reported pacing lead longevity studies in children and young adults. Both the SelectSecure and conventional pacing lead groups demonstrated excellent electrical function but the SelectSecure pacing lead group did exhibit slightly higher capture thresholds and increased calculated energy to pace over time.

\section{References}

1. Olgun $\mathrm{H}$, Karagoz T, Celiker A, Ceviz N. Patient- and lead-related factors affecting lead fracture in children with transvenous permanent pacemaker. Europace. 2008; 10:844-847.

2. Fortescue EB, Berul Cl, Cecchin F, Walsh EP, Triedman JK, Alexander ME. Patient, procedural, and hardware factors associated with pacemaker lead failures in pediatrics and congenital heart disease. Heart Rhythm. 2004; 1:150-159.

3. Silvetti MS, Drago F, De Santis A, Grutter G, Ravà L, Monti L, Fruhwirth R. Single-centre experience on endocardial and epicardial pacemaker system function in neonates and infants. Europace; 2007; 9:426-431.

4. Lau YR, Gillette PC, Buckles DS, Zeigler VL. Actuarial survival of transvenous pacing leads in a pediatric population. Pacing Clin Electrophysiol. 1993; 16:1363-1367.

5. Stojanov PL, Savic DV, Zivkovic MB, Calovic ZR. Permanent endovenous pediatric pacing: absence of lead failure--20 years follow-up study. Pacing Clin Electrophysiol. 2008; 3:1100-1107.

6. Beaufort-Krol GC, Mulder H, Nagelkerke D, Waterbolk TW, Bink-Boelkens MT. Comparison of longevity, pacing, and sensing characteristics of steroid-eluting epicardial versus conventional endocardial pacing leads in children. J Thorac Cardiovasc Surg. 1999; 7:523-528.

7. Cecchin F, Atallah J, Walsh EP, Triedman JK, Alexander ME, Berul CI. Lead extraction in pediatric and congenital heart disease patients. Circ Arrhythm Electrophysiol. 2010; 3:437-444.

This article is protected by copyright. All rights reserved. 
8. Shepherd E, Stuart G, Martin R, Walsh MA. Extraction of SelectSecure leads compared to conventional pacing leads in patients with congenital heart disease and congenital atrioventricular block. Heart Rhythm. 2015; 12:1227-1232.

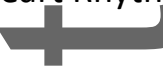

9. ChakrabartiS, Morgan GJ, Kenny D, Walsh KP, Oslizlok P, Martin RP, Turner MS, et al. Initial experience of pacing with a lumenless lead system in patients with congenital heart disease. Pacing Clin Electrophysiol. 2009; 32:1428-1433.

10. StataCorp. 2017. Stata Statistical Software: Release 15. College Station, TX: StataCorp LLC

11. Garnreiter J, Whitaker P, Pilcher T, Etheridge S, Saarel E. Lumenless pacing leads: performance and extraction in pediatrics and congenital heart disease. Pacing Clin Electrophysiol. 2015; 38:42-47.

12. Gammage MD, Lieberman RA, Yee R, Manolis AS, Compton SJ, Khazen C, Schaaf K, et al. Multi-center clinical experience with a lumenless, catheter-delivered, bipolar, permanent pacemaker lead: implant safety and electrical performance. Pacing Clin Electrophysiol. 2006; 29:858-865.

13. Bansal N, Samuel S, Zelin K, Karpawich PP. Ten-Year Clinical Experience with the Lumenless, Catheter-Delivered, 4.1-Fr Diameter Pacing Lead in Patients with and without Congenital Heart. Pacing Clin Electrophysiol. 2017; 40:17-25.

14. Cantù F, De Filippo $P$, Gabbarini $F$, Borghi $A$, Brambilla R, Ferrero $P$, Comisso $\mathrm{J}$, et al. Selective-site pacing in paediatric patients: a new application of the Select Secure system. Europace. 2009; 11:601-606.

15. De Filippo $P$, Giofrè F, Leidi $C$, Senni M, Ferrari P. Transvenous pacing in pediatric patients with bipolar lumentess lead: Ten-year clinical experience. Int J Cardiol. 2018 Mar 15;255:45-49. doi: 10.1016/j.ijcard.2018.01.007. Epub 2018 Jan 4.

16. Khan A, Zelin K, Karpawich PP. Performance of the lumenless 4.1-Fr diameter pacing lead implanted at alternative pacing sites in congenital heart: a chronic 5-year comparison. Pacing Clin Electrophysiol. 2010; 33:1467-1474.

17. Gelvan D, Crystal E, Dokumaci B, Goldshmid Y, Ovsyshcher IE. Effect of modern pacing algorithms on generator longevity: a predictive analysis. Pacing Clin Electrophysiol. 2003; 26: 1796-1802.

18. Cohen MI, Buek K, Tanel RE, Vetter VL, Rhodes LA, Cox J, Sheldon T, Ruetz L. Capture management efficacy in children and young adults with endocardial and unipolar epicardial systems. Europace. 2004; 6:248-255.

This article is protected by copyright. All rights reserved. 
19. Karpawich PP, Singh H, Zelin K. Optimizing paced ventricular function in patients with and without repaired congenital heart disease by contractility-guided lead implant. Pacing Clin Electrophysiol. 2015; 38:54-62.

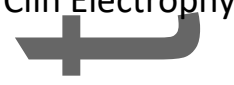

20. Sharma PS, Dandamudi G, Herweg B, Wilson D, Singh R, Naperkowski

A, Koneru JN, et al. Permanent His-bundle pacing as an alternative to biventricular pacing for cardiac resynchronization therapy: A multicenter experience. Heart Rhythm. 2018; 15:413-420.

21. Redfearn DP, Gula L, Krahn AD, Skanes AC, Klein GJ, Yee R. Current of injury predicts acute performance of catheter-delivered active fixation pacing leads.

Pacing Clin Electrophysiol. 2007; 3:1438-1444.

22. Shali S, Wushou A, Liu E, Jia L, Yao R, Su Y, Ge J. Time course of current of injury is related to acute stability of active-fixation pacing leads in rabbits. PLoS One. 2013;8(3):e57727. doi: 10.1371/journal.pone.0057727. Epub 2013 Mar 5.

23. Bharmanee A, Zelin K, Sanil Y, Gupta P, Karpawich PP. Comparative Chronic Valve and Venous Effects of Lumenless versus Stylet-Delivered Pacing Leads in Patients with and Without Congenital Heart. Pacing Clin Electrophysiol. 2015; 38:1343-1350.

Figure Legends

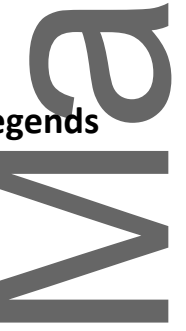

Figure 1: Longitudinal changes in capture threshold to pace for the atrial and ventricular pacing leads (Volts). A. Longitudinal changes in atrial lead capture thresholds. Atrial capture threshold was higher in the SelectSecure pacing group, $p<0.001$. B. Longitudinal changes in ventricular lead capture thresholds. Ventricular capture threshold was higher in the SelectSecure pacing group, $<0.001$. SelectSecure pacing lead data are illustrated in red, and conventional pacing leads in blue. Line represents median data.

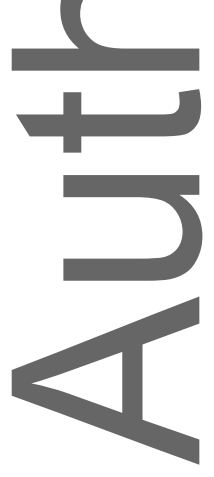

This article is protected by copyright. All rights reserved. 

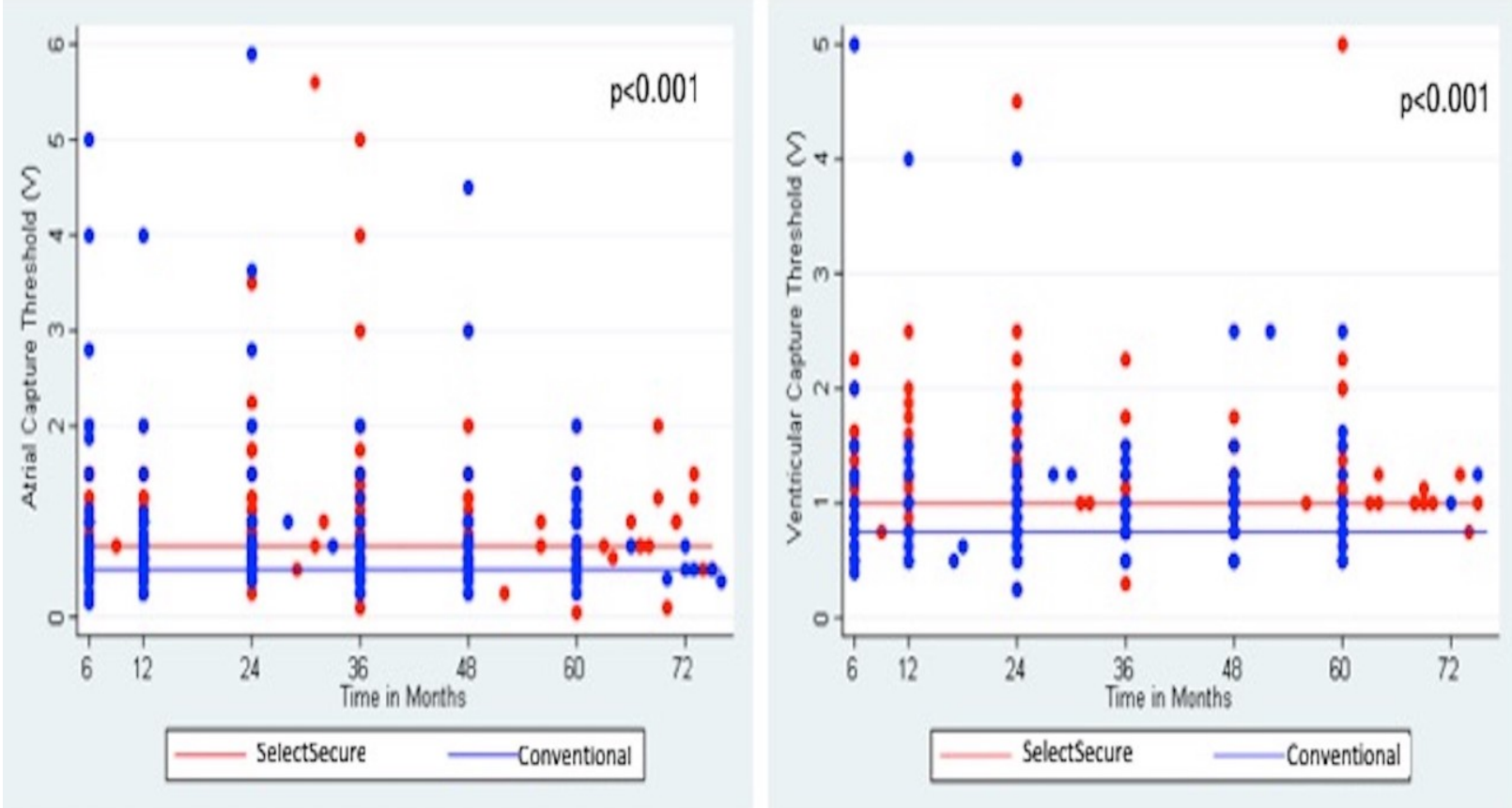

A.

B.

Figure 1.

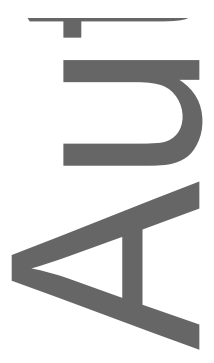

This article is protected by copyright. All rights reserved. 
Figure 2: Longitudinal changes in atrial and ventricular pacing lead impedance (Ohms).

A. Atrial leads. Atrial pacing lead impedance was higher in the SelectSecure pacing group, $p<0.016$. B. Ventricular leads. Ventricular pacing lead impedance was higher in the SelectSecure pacing group, $p=0.045$. SelectSecure pacing lead data are illustrated in red, and conventional pacing leads in blue. Line represents median data.
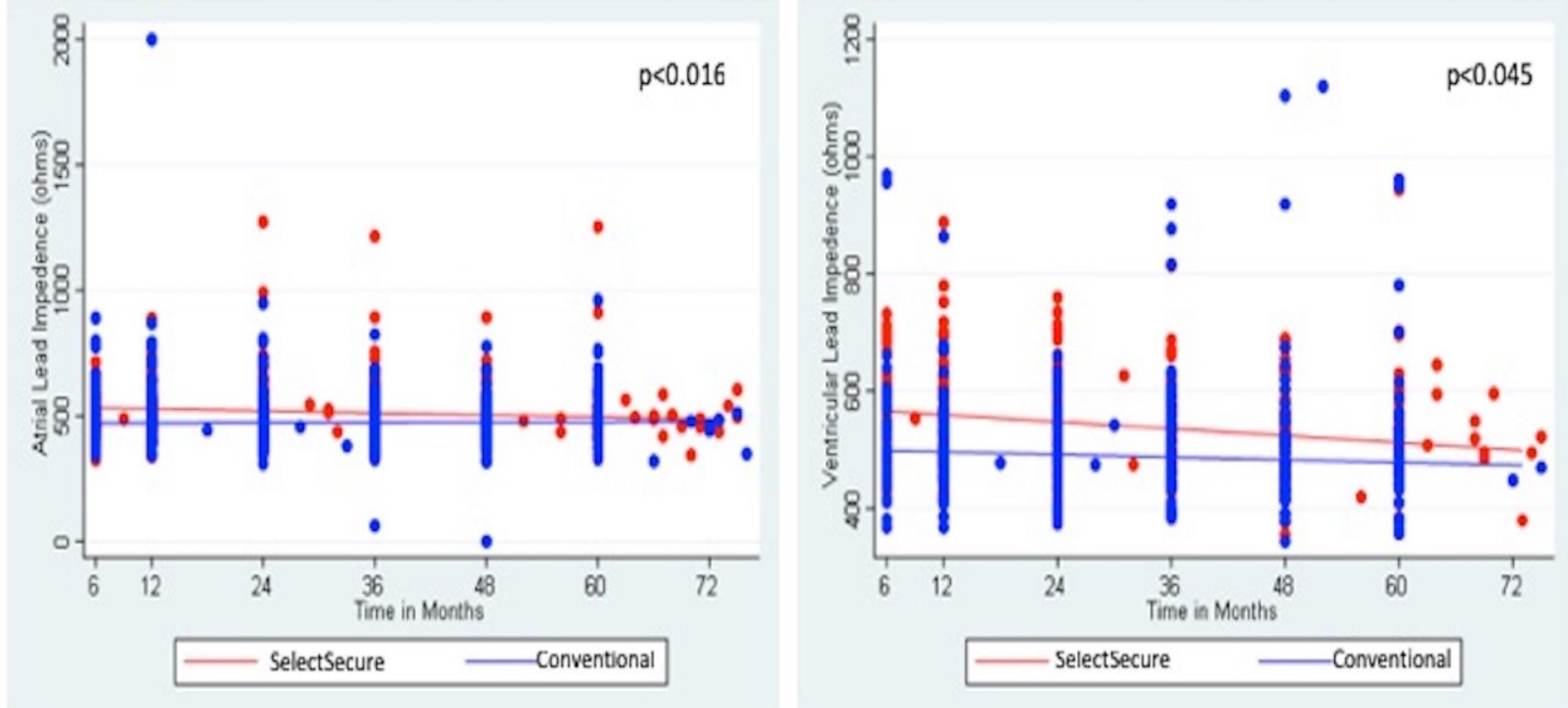

A.

B.

Figure 2.

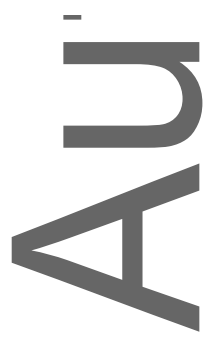

This article is protected by copyright. All rights reserved. 
Figure 3: Longitudinal changes in threshold energy to pace for the atrial and ventricular pacing leads (mJ). A. Atrial leads. Atrial lead energy to pace was higher in the SelectSecure pacing group, $p<0.001$. B. Ventricular leads. Ventricular lead energy to pace was higher in the SelectSecure pacing group, $p=0,001$. SelectSecure pacing lead data are illustrated in red, and conventional pacing leads in blue. Line represents median data.

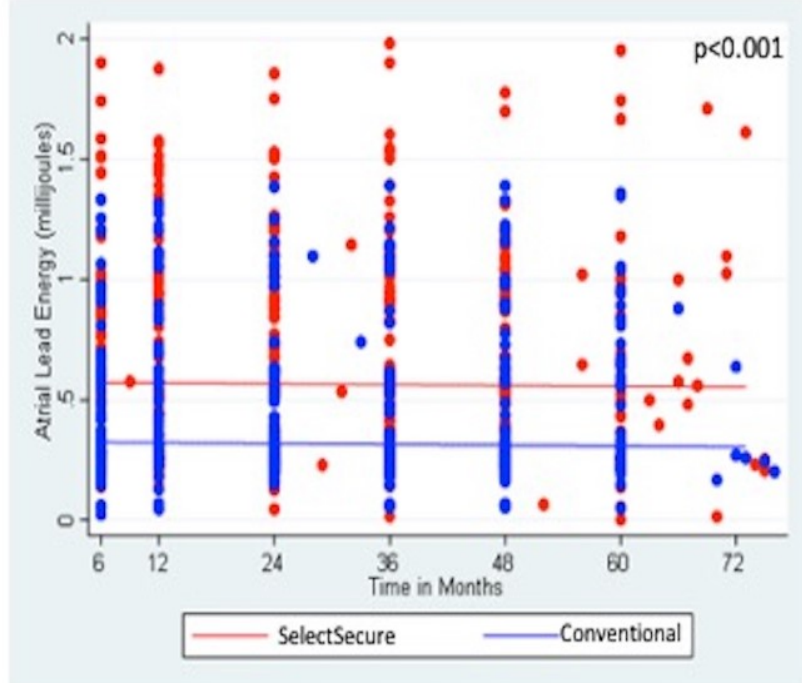

A.

Figure 3.

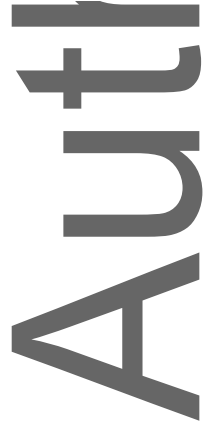

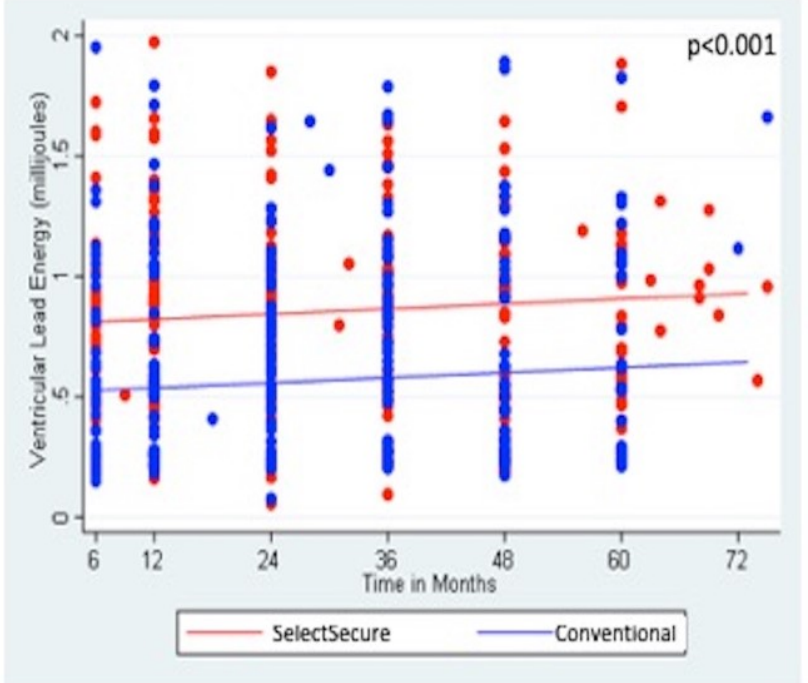

B.

This article is protected by copyright. All rights reserved. 
Figure 4: Kaplan-Meier plot of pacing lead survival free from complications. SelectSecure pacing lead data are illustrated in red and conventional pacing lead data are shown in black. No significant difference noted between the two groups up to 72 months of follow-up, $p=0.50$. Number of leads at risk provided at bottom of graph.

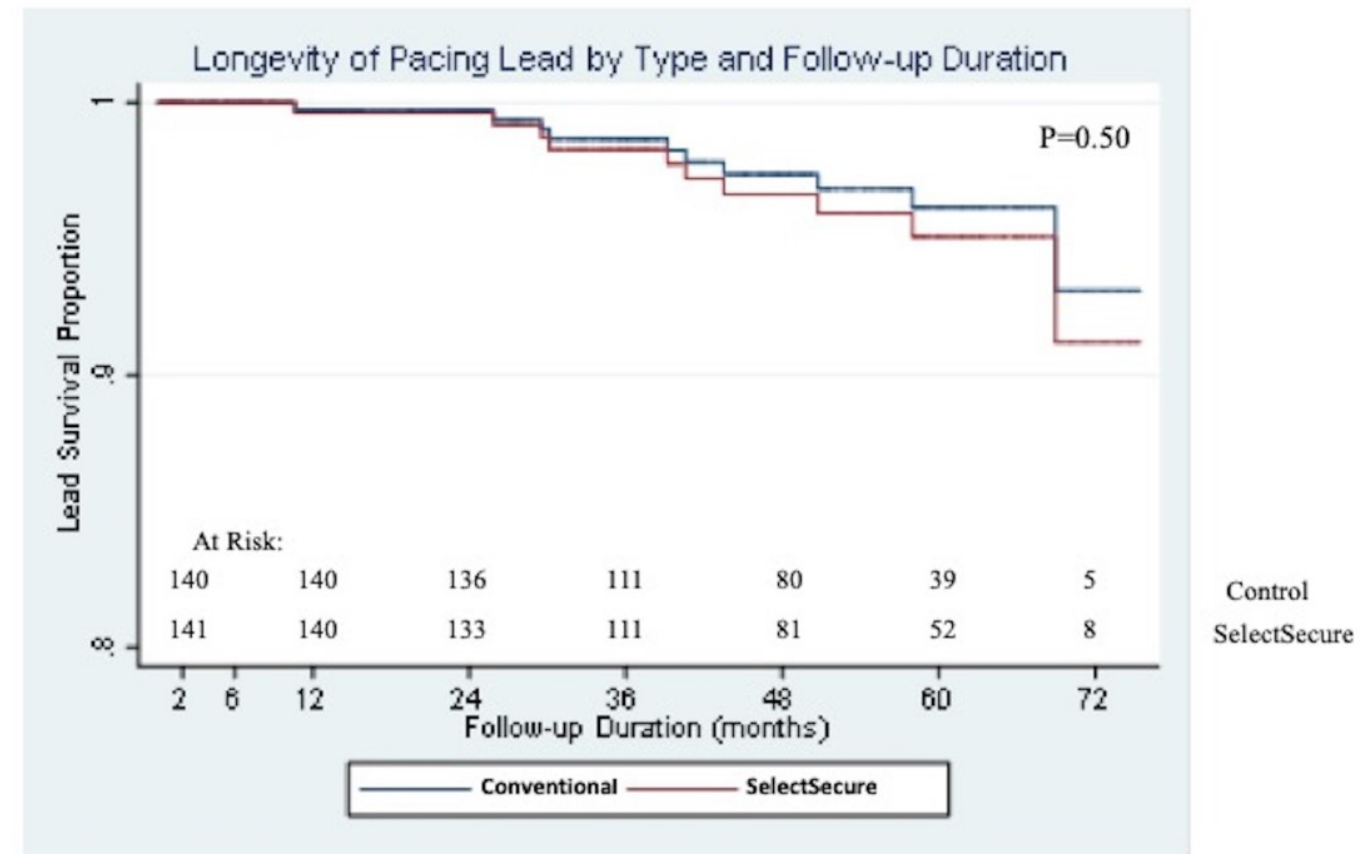

Figure 4.

Table 1: Manufacturer and Pacing Lead Model Numbers Implanted in the Conventional Pacing Lead Group

Conventional Pacing Leads

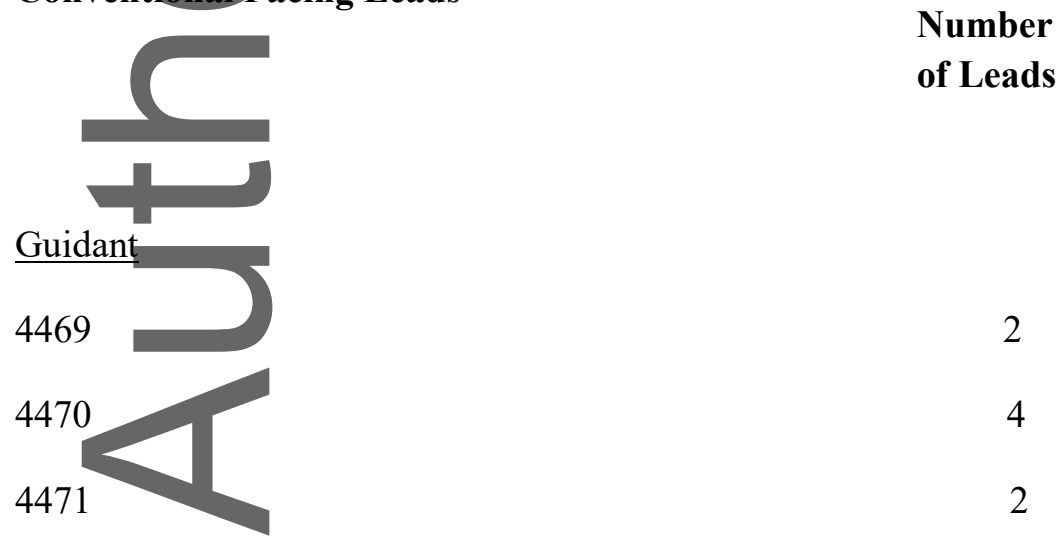

This article is protected by copyright. All rights reserved. 

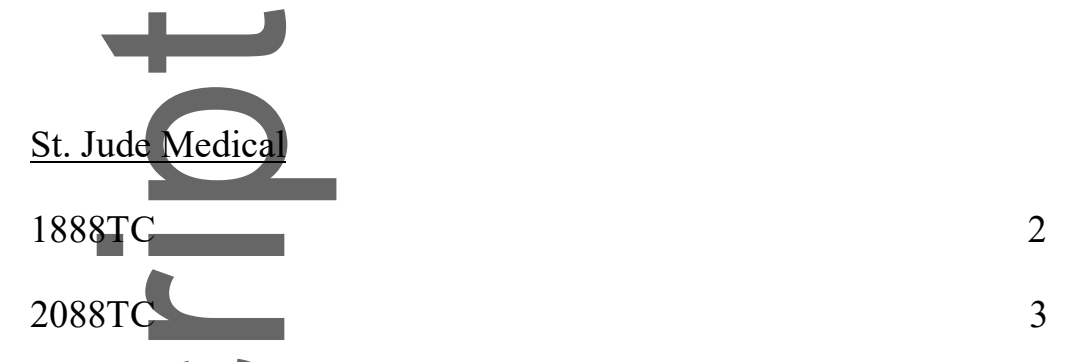

(C)

Medtronic

4076

47

5076

76

3

Table 2: Demographic Characteristics of the SelectSecure and Conventional Pacing Lead Participants
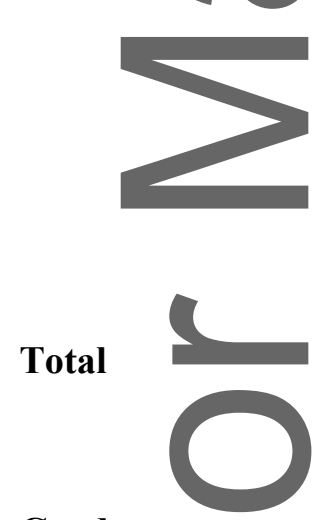

Gender

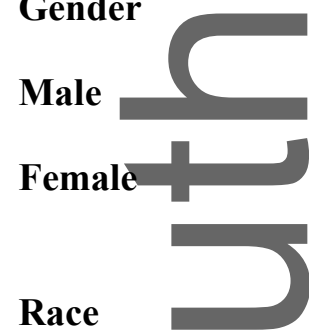

American Indian/Alaska Native

Asian

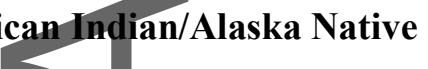

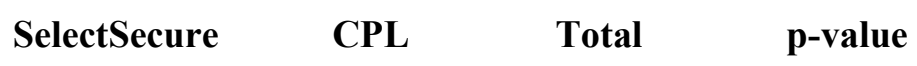

$\begin{array}{llllll}\mathbf{N} & \% & \mathbf{N} & \% & \mathbf{N} & \%\end{array}$

$\begin{array}{llllll}141 & 100 & 141 & 100 & 282 & 100\end{array}$

$\begin{array}{llllll}84 & 59.6 & 71 & 50.4 & 155 & 55\end{array}$

$\begin{array}{llllll}57 & 40.4 & 70 & 49.6 & 127 & 45\end{array}$

0.77

$\begin{array}{llllll}0 & 0.0 & 1 & 0.7 & 1 & 0.4 \\ 2 & 1.4 & 3 & 2.1 & 5 & 1.8\end{array}$

26

This article is protected by copyright. All rights reserved. 


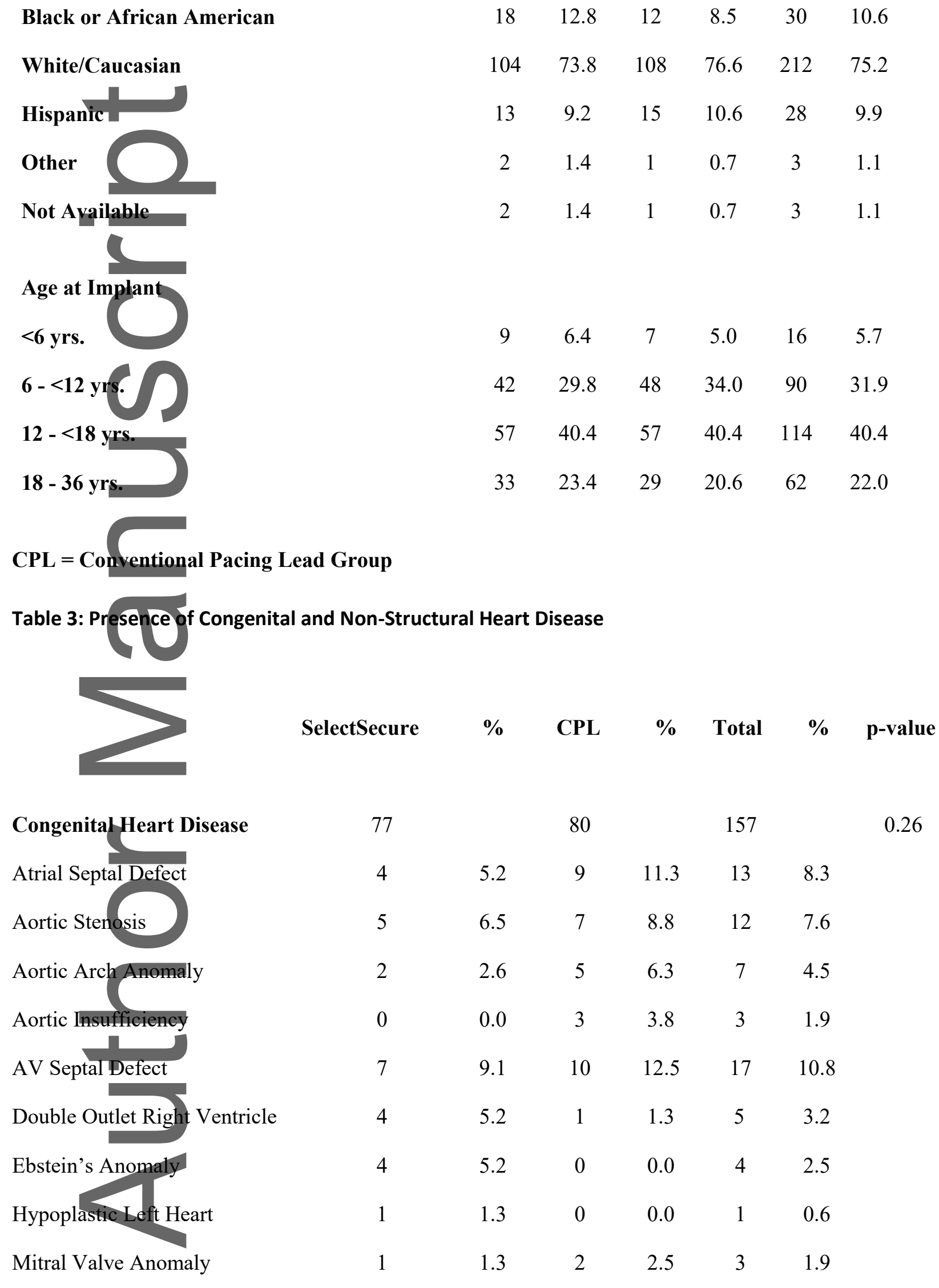

This article is protected by copyright. All rights reserved. 


\begin{tabular}{|c|c|c|c|c|c|c|c|}
\hline Pulmonary Artery Atresia & 2 & 2.6 & 0 & 0.0 & 2 & 1.3 & \\
\hline Pulmonary Stenosis & 0 & 0.0 & 1 & 1.3 & 1 & 0.6 & \\
\hline Single Ventricle & 1 & 1.3 & 1 & 1.3 & 2 & 1.3 & \\
\hline Tetralogy of Fallot & 14 & 18.2 & 8 & 10.0 & 22 & 14.0 & \\
\hline Total Anomalous PV Return & 4 & 5.2 & 4 & 5.0 & 8 & 5.1 & \\
\hline Tricuspid $A$ & 1 & 1.3 & 0 & 0.0 & 1 & 0.6 & \\
\hline Truncus & 1 & 1.3 & 2 & 2.5 & 3 & 1.9 & \\
\hline Ventricular Septal Defect & 7 & 9.1 & 9 & 11.3 & 16 & 10.2 & \\
\hline D-TGA & 12 & 15.6 & 14 & 17.5 & 26 & 16.6 & \\
\hline L-TGA & 7 & 9.1 & 4 & 5.0 & 11 & 7.0 & \\
\hline Cardiomyop: & 14 & & 15 & & 29 & & 1.00 \\
\hline Dilated & 6 & 42.9 & 7 & 46.7 & 13 & 44.8 & \\
\hline Hypertrop & 6 & 42.9 & 7 & 46.7 & 13 & 44.8 & \\
\hline Other & 2 & 14.3 & 1 & 6.7 & 3 & 10.3 & \\
\hline Acquired-Heart Disease & 6 & & 2 & & 8 & & 0.68 \\
\hline Myocarditis & 0 & 0.0 & 1 & 50.0 & 1 & 12.5 & \\
\hline Cardiac Transpl & 3 & 50.0 & 0 & 0.0 & 3 & 37.5 & \\
\hline Infectious Endocarditis & 1 & 16.7 & 0 & 0.0 & 1 & 12.5 & \\
\hline Other & 2 & 33.3 & 1 & 50.0 & 3 & 37.5 & \\
\hline
\end{tabular}

AV = atrioventricular, CPL $=$ Conventional Pacing Lead Group,

TGA $=$ transposition of great arteries

Table 4: Indications for Pacing

$\begin{array}{ccccccr}\text { SelectSecure } & \% & \text { CPL } & \% & \text { Total } & \% & \text { p-value } \\ 165 & & 148 & & 313 & & 0.39\end{array}$

Implant Indications

28

This article is protected by copyright. All rights reserved. 
Sick Sinus Syndrome (Tachy-Brady

Syndrome)

Sinus Node Dysfunction / Sinus Pauses

Second Degree AK Block

Complete AV Block

Atrial Rhythm detection for DDD ICD

Other

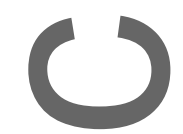

CPL = Conventional Pacing Lead Group

ICD = implantable cardioverter defibrillator, DDD = dual chamber $\begin{array}{llllll}21 & 12.7 & 11 & 7.4 & 32 & 10.2\end{array}$

$\begin{array}{llllll}33 & 20 & 30 & 20.3 & 63 & 20.1\end{array}$

$\begin{array}{llllll}22 & 13.3 & 17 & 11.5 & 39 & 12.5\end{array}$

$\begin{array}{llllll}62 & 37.6 & 62 & 41.9 & 124 & 39.6\end{array}$

$\begin{array}{llllll}23 & 13.9 & 19 & 12.8 & 42 & 13.4\end{array}$

$\begin{array}{llllll}4 & 2.4 & 9 & 6.1 & 13 & 4.2\end{array}$

Table 5: Complications Reported Greater than 1 Month Following Pacing Lead Implantation

Type of Complication

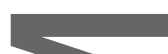

Total Participants Reporting a

Complication

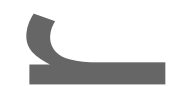

Total Complications Reported

Type of Complication

Failure to Capture

Lead Fracture

Significant Increase in Capture

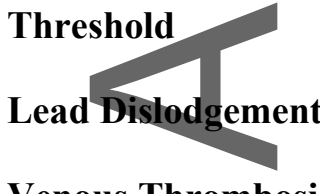

Venous Thrombosis
SelectSecure

CPL

Total

$\begin{array}{llllll}\mathbf{N} & \% & \mathbf{N} & \% & \mathbf{N} & \%\end{array}$

6

4.4

9

6.4

15

8

5.8

10

7.2

18

6.4

2

1.4

3

2.1

5

1.9

2

1.4

2

1.4

1.4

1

0.7

0

0.0

0.3

3

2.10

0.0

1.1

0

0.0

2

1.4

0.7 


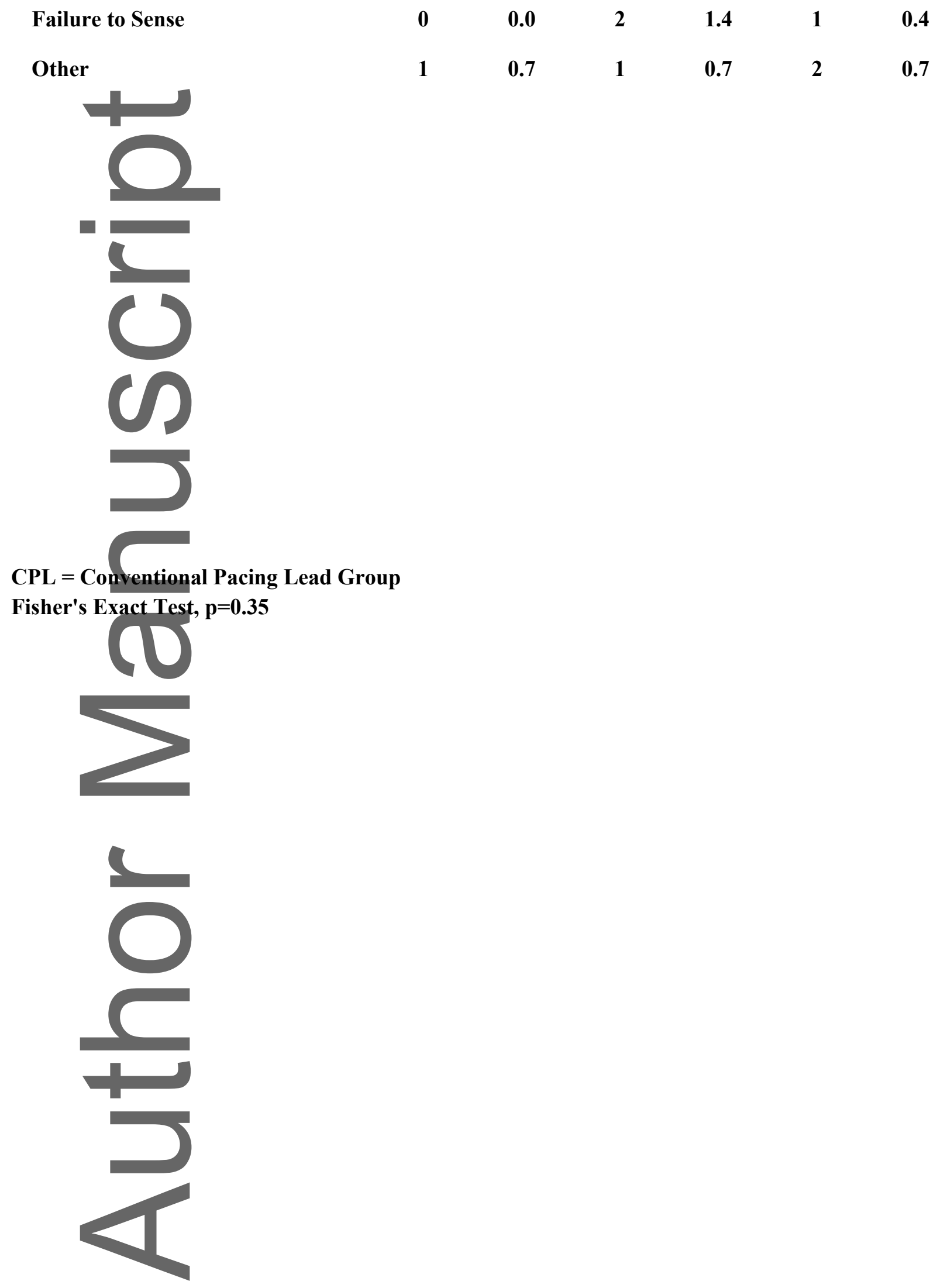

This article is protected by copyright. All rights reserved. 